Supporting Information for the Communication:

\title{
Dehydrogenative Coupling of 4-Substituted Pyridines Catalyzed by Diruthenium Complexes.
}

Takashi Kawashima, Toshiro Takao, and Hiroharu Suzuki*

Department of Applied Chemistry, Graduated School of Science and Engineering

Tokyo Institute of Technology, 2-12-1 O-okayama, Meguro-ku, Tokyo 152-8552, Japan

General Procedures. All compounds were manipulated using standard Schlenk and high-vacuum line techniques under an argon atmosphere. Dehydrated tetrahydrofuran used in this study was purchased from Kanto Chemicals and stored under an atmosphere of argon. Pyridines and decane were dried over molecular sieves 4A and stored under an argon atmosphere. Mesitylene, tetrahydrofuran- $d_{8}$, and benzene- $d_{6}$ were dried over sodium-benzophenone ketyl and stored under an argon atmosphere. Other reagents were used as received. A diruthenium tetrahydrido complex, $\left(\mathrm{Cp}^{*} \mathrm{Ru}\right)_{2}(\mu-\mathrm{H})_{4}\left(\mathbf{1} ; \mathrm{Cp}^{*}=\right.$ $\eta^{5}-\mathrm{C}_{5} \mathrm{Me}_{5}$ ), was prepared according to the previously published methods. ${ }^{1} \mathrm{~A}$ diruthenium $\mu$-phosphido complex, $(\mathrm{Cp} * \mathrm{Ru})_{2}\left(\mu-\mathrm{PMe}_{2}\right)\left(\mu-\eta^{2}: \eta^{2}-\mathrm{C}_{6} \mathrm{H}_{6}\right)(\mu-\mathrm{H})(2){ }^{2}$ were prepared by the similar method to the preparation of diphenylphosphido analogue using $\mathrm{PPhMe}_{2}$ instead of $\mathrm{PPh}_{3}$. The ${ }^{1} \mathrm{H},{ }^{13} \mathrm{C}$, and ${ }^{31} \mathrm{P}$ NMR spectra were recorded on Varian INOVA-400 and GEMINI-300 spectrometers. ${ }^{1} \mathrm{H}$ NMR spectra were referenced to tetramethylsilane as an internal standard. ${ }^{13} \mathrm{C}$ NMR spectra were referenced to the natural-abundant ${ }^{13} \mathrm{C}$ signal of the solvent employed. ${ }^{31} \mathrm{P}$ NMR spectra were referenced to external phosphoric acid. Elemental analysis was performed on a Perkin Elmer 2400II. GLC analyses were performed on a SHIMADZU GC-17A using a capillary column (J\&W DB-1; $30 \mathrm{~m} \times 0.53 \mathrm{~mm} \times 1.50 \mu \mathrm{m})$ with helium gas as a carrier. 
[2] Omori, H.; Suzuki, H.; Moro-oka, Y. Organometallics 1989, 8, 2270.

X-ray Diffraction Studies. Single crystals of $\mathbf{3}^{\cdot} \mathbf{C}_{5} \mathbf{H}_{\mathbf{1 2}}$ and $\mathbf{5}$ for an X-ray analysis were obtained directly from the preparations described below and mounted on glass fibers. Diffraction experiments were performed on a Rigaku R-AXIS RAPID imaging plate Diffractometer with graphite-monochromated Mo K $\alpha$ radiation $(\lambda=0.71069 \AA)$. In all samples, cell refinement and data reduction were performed using the PROCESS-AUTO program. ${ }^{3}$ Intensity data were corrected for Lorentz-polarization effects and for empirical absorption. The structures of $\mathbf{3} \cdot \mathbf{C}_{5} \mathbf{H}_{12}$ and $\mathbf{5}$ were solved by the Patterson method using SHELX-97 program package. ${ }^{4}$ All non-hydrogen atoms were found by the difference Fourier synthesis and were refined anisotropically except for the disordered atoms described below. The refinement was carried out by least-squares methods based on $F^{2}$ with all measured reflections. For $\mathbf{3} \cdot \mathbf{C}_{5} \mathbf{H}_{12}$, disorder at all atoms were refined in the ratio of $50: 50$. For 5 , The metal-bound hydrogen atoms of 5 was located in difference Fourier map and refined isotropically. Crystal data and results of the analyses are listed in Table S-1 and S-2.

[3] PROCESS-AUTO, Automatic Data Acquisition and Processing Package for Imaging Plate Diffractometer; Rigaku Corporation: Tokyo (Japan), 1998.

[4] G. M. Sheldrick, SHELX-97, Program for Crystal Structure Determination; University of Göttingen: Göttingen (Germany), 1997. 
Table S-1. Crystallographic Data for $\mathbf{3} \cdot \mathbf{C}_{5} \mathbf{H}_{\mathbf{1 2}}$.

\begin{tabular}{|c|c|c|c|}
\hline \multicolumn{2}{|l|}{ (a) Crystal Data } & \multicolumn{2}{|l|}{ (b) Intensity Measurements } \\
\hline Empirical formula & $\mathrm{C}_{32} \mathrm{H}_{44} \mathrm{~N}_{2} \mathrm{O}_{2} \mathrm{Ru}_{2} \cdot \mathrm{C}_{5} \mathrm{H}_{12}$ & Diffractometer & R-AXIS RAPID \\
\hline Formula weight & 762.98 & Radiation & $\operatorname{Mo} \mathrm{K} \alpha(\lambda=0.71069 \AA)$ \\
\hline Crystal description & Prism & Monochromator & Graphite \\
\hline Crystal color & Yellow & $\theta$ range for data collection & $3.02-27.46^{\circ}$ \\
\hline Crystal size (mm) & $0.29 \times 0.18 \times 0.09$ & Index ranges & $-13 \leq h \leq 12$ \\
\hline Crystallizing solution & Pentane $\left(-20^{\circ} \mathrm{C}\right)$ & & $-17 \leq k \leq 17$ \\
\hline Crystal system & Monoclinic & & $-17 \leq l \leq 17$ \\
\hline Space group & $P 2_{1} / m(\# 11)$ & No. of reflections collected & 17165 \\
\hline$a(\AA)$ & $10.1058(4)$ & No. of unique reflections & $4283\left(R_{\mathrm{int}}=0.0295\right)$ \\
\hline$b(\AA)$ & $13.1552(6)$ & Abs. correction type & Empirical \\
\hline$c(\AA)$ & $13.5335(5)$ & Abs. transmission & 0.6159 (min.), 1.0000 (max.) \\
\hline$\alpha\left({ }^{\circ}\right)$ & & (c) Refinement (SHELXL-97) & \\
\hline$\beta\left(^{\circ}\right)$ & $93.5110(11)$ & $R_{1}[I>2 \sigma(I)]$ & 0.0501 \\
\hline$\gamma\left({ }^{\circ}\right)$ & & $w R_{2}[I>2 \sigma(I)]$ & 0.1251 \\
\hline$V\left(\AA^{3}\right)$ & $1795.82(13)$ & $R_{1}$ (all data) & 0.0652 \\
\hline$Z$ & 2 & $w R_{2}$ (all data) & 0.1386 \\
\hline$\rho_{\text {calcd }}\left(\mathrm{Mg} / \mathrm{m}^{3}\right)$ & 1.411 & Data / restraints / parameters & 4264 / 0 / 349 \\
\hline Measurement temp. $\left({ }^{\circ} \mathrm{C}\right)$ & -120 & Goodness of fit on $F^{2}$ & 1.111 \\
\hline$\mu\left(\mathrm{Mo}_{\mathrm{K} \alpha}\right)\left(\mathrm{mm}^{-1}\right)$ & 0.874 & Largest diff. peak and hole & 0.893 and $-0.406 \mathrm{e} \cdot \AA^{-3}$ \\
\hline
\end{tabular}

Table S-2. Crystallographic Data for 5

\begin{tabular}{|c|c|c|c|}
\hline \multicolumn{2}{|l|}{ (a) Crystal Data } & \multicolumn{2}{|l|}{ (b) Intensity Measurements } \\
\hline Empirical formula & $\mathrm{C}_{32} \mathrm{H}_{45} \mathrm{~N}_{2} \mathrm{PRu}_{2}$ & Diffractometer & R-AXIS RAPID \\
\hline Formula weight & 690.81 & Radiation & $\operatorname{Mo~} \mathrm{K} \alpha(\lambda=0.71069 \AA)$ \\
\hline Crystal description & Platelet & Monochromator & Graphite \\
\hline Crystal color & Red & $\theta$ range for data collection & $2.99-27.47^{\circ}$ \\
\hline Crystal size (mm) & $0.12 \times 0.05 \times 0.02$ & Index ranges & $-11 \leq h \leq 11$ \\
\hline Crystallizing solution & Pentane $\left(2{ }^{\circ} \mathrm{C}\right)$ & & $-12 \leq k \leq 12$ \\
\hline Crystal system & Triclinic & & $-23 \leq l \leq 23$ \\
\hline Space group & $P-1(\# 2)$ & No. of reflections collected & 13843 \\
\hline$a(\AA)$ & $9.0276(6)$ & No. of unique reflections & $6854\left(R_{\mathrm{int}}=0.0364\right)$ \\
\hline$b(\AA)$ & $9.6462(5)$ & Abs. correction type & Empirical \\
\hline$c(\AA)$ & $18.1425(9)$ & Abs. transmission & 0.7158 (min.), 1.0000 (max.) \\
\hline$\alpha\left(^{\circ}\right)$ & $96.3139(15)$ & (c) Refinement (SHELXL-97) & \\
\hline$\beta\left(\left(^{\circ}\right)\right.$ & $94.4097(18)$ & $R_{1}[I>2 \sigma(I)]$ & 0.0347 \\
\hline$\gamma\left({ }^{\circ}\right)$ & $103.3716(19)$ & $w R_{2}[I>2 \sigma(I)]$ & 0.0743 \\
\hline$V\left(\AA^{3}\right)$ & $1519.16(15)$ & $R_{1}$ (all data) & 0.0478 \\
\hline$Z$ & 2 & $w R_{2}$ (all data) & 0.0800 \\
\hline$\rho_{\text {calcd }}\left(\mathrm{Mg} / \mathrm{m}^{3}\right)$ & 1.510 & Data / restraints / parameters & 17649 / 1 / 649 \\
\hline Measurement temp. $\left({ }^{\circ} \mathrm{C}\right)$ & -100 & Goodness of fit on $F^{2}$ & 1.033 \\
\hline$\mu\left(\mathrm{Mo}_{\mathrm{K} \alpha}\right)\left(\mathrm{mm}^{-1}\right)$ & 1.070 & Largest diff. peak and hole & 0.877 and $-0.676 \mathrm{e} \cdot \AA^{-3}$ \\
\hline
\end{tabular}


Results of the X-ray diffraction studies of $\left(\mathrm{Cp}^{*} \mathrm{Ru}\right)_{2}\left\{\mu-\mathrm{C}_{5} \mathrm{H}_{3}(\mathrm{OMe}) \mathrm{N}\right\}(\mu-\mathrm{H})_{2}(3)$.

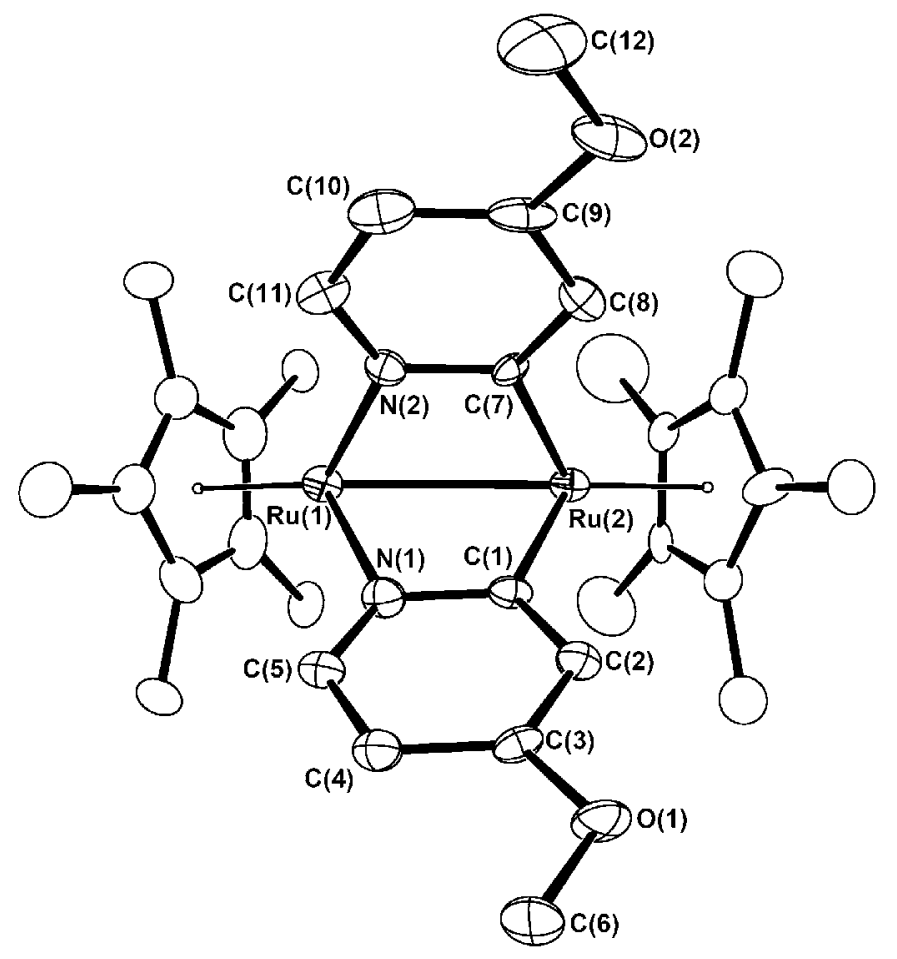

Table S-3. Selected bond lengths $(\AA)$ and angles $\left({ }^{\circ}\right)$ for 3

\begin{tabular}{llllll}
\hline Ru1-Ru2 & $2.8666(10)$ & Ru1-N1 & $2.112(7)$ & Ru1-N2 & $2.113(7)$ \\
Ru2-C1 & $2.052(7)$ & Ru2-C7 & $2.063(8)$ & N1-C1 & $1.389(9)$ \\
C1-C2 & $1.395(11)$ & C2-C3 & $1.388(10)$ & C3-C4 & $1.420(10)$ \\
C4-C5 & $1.380(11)$ & N1-C5 & $1.329(10)$ & N2-C7 & $1.368(9)$ \\
C7-C8 & $1.413(14)$ & C8-C9 & $1.37(2)$ & C9-C10 & $1.42(2)$ \\
C10-C11 & $1.39(2)$ & N2-C11 & $1.342(13)$ & & \\
& & & & & \\
Ru1-N1-C1 & $108.6(4)$ & Ru1-N1-C5 & $131.1(6)$ & Ru1-N2-C7 & $109.5(4)$ \\
Ru1-N2-C11 & $130.6(6)$ & Ru2-C1-N1 & $112.9(4)$ & Ru2-C1-C2 & $129.5(6)$ \\
Ru2-C7-N2 & $112.6(5)$ & Ru2-C7-C 8 & $128.1(6)$ & C1-N1-C5 & $120.1(6)$ \\
N1-C1-C2 & $117.5(6)$ & C1-C2-C3 & $121.6(7)$ & C2-C3-C4 & $119.8(6)$ \\
C3-C4-C5 & $115.5(7)$ & N1-C5-C4 & $125.4(9)$ & C7-N2-C11 & $119.9(7)$ \\
N2-C7-C & $118.8(7)$ & C7-C8-C & $120.2(8)$ & C8-C9-C10 & $120.8(12)$ \\
C9-C10-C11 & $115.5(13)$ & N2-C11-C10 & $124.4(10)$ & & \\
\hline
\end{tabular}


Results of the X-ray diffraction studies of $\left(C p^{*} R u\right)_{2}\left(\mu-\eta^{2}: \eta^{2}-2,2^{\prime}-b p y\right)-$ $\left(\mu-\mathrm{PMe}_{2} \mathrm{H}\right)(\mu-\mathrm{H})(5)$.

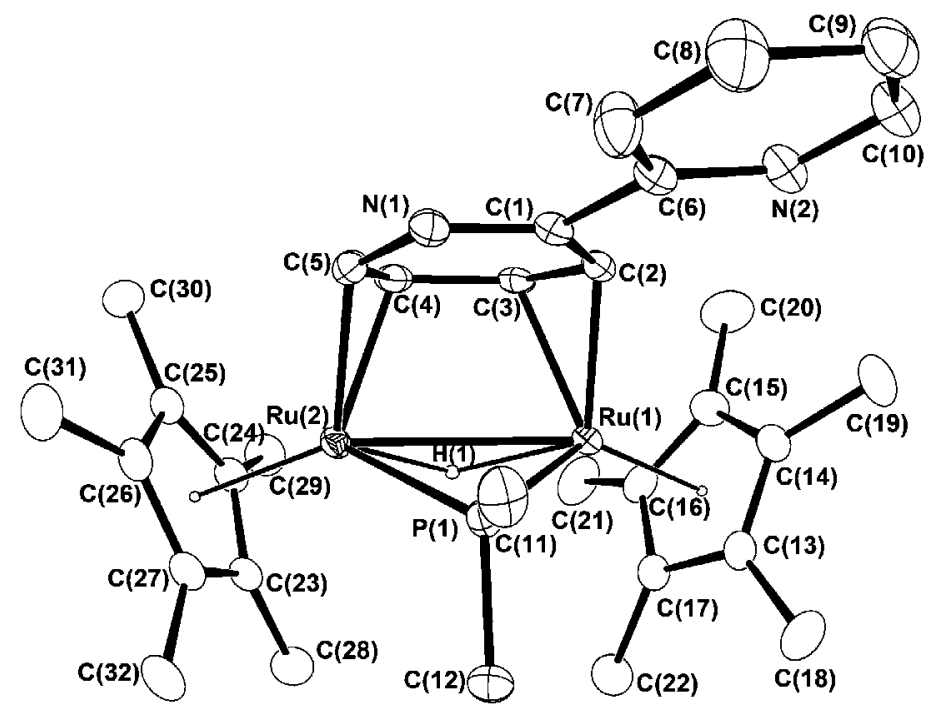

Table S-4. Selected bond lengths $(\AA)$ and angles $\left({ }^{\circ}\right)$ for 5

\begin{tabular}{llllll}
\hline Ru1-Ru2 & $2.9231(3)$ & $\mathrm{Ru} 1-\mathrm{P} 1$ & $2.2955(8)$ & $\mathrm{Ru} 2-\mathrm{P} 1$ & $2.2924(7)$ \\
$\mathrm{Ru} 1-\mathrm{C} 2$ & $2.202(3)$ & $\mathrm{Ru} 1-\mathrm{C} 3$ & $2.169(3)$ & $\mathrm{Ru} 2-\mathrm{C} 4$ & $2.151(3)$ \\
$\mathrm{Ru} 2-\mathrm{C} 5$ & $2.213(3)$ & $\mathrm{N} 1-\mathrm{C} 1$ & $1.307(4)$ & $\mathrm{C} 1-\mathrm{C} 2$ & $1.444(4)$ \\
$\mathrm{C} 2-\mathrm{C} 3$ & $1.429(4)$ & $\mathrm{C} 3-\mathrm{C} 4$ & $1.454(5)$ & $\mathrm{C} 4-\mathrm{C} 5$ & $1.429(5)$ \\
$\mathrm{N} 1-\mathrm{C} 5$ & $1.409(4)$ & & & & \\
& & & & & \\
$\mathrm{Ru} 1-\mathrm{P} 1-\mathrm{Ru} 2$ & $79.16(2)$ & $\mathrm{C} 1-\mathrm{N} 1-\mathrm{C} 5$ & $117.2(3)$ & $\mathrm{N} 1-\mathrm{C} 1-\mathrm{C} 2$ & $124.6(3)$ \\
$\mathrm{C} 1-\mathrm{C} 2-\mathrm{C} 3$ & $117.9(3)$ & $\mathrm{C} 2-\mathrm{C} 3-\mathrm{C} 4$ & $118.0(3)$ & $\mathrm{C} 3-\mathrm{C} 4-\mathrm{C} 5$ & $116.9(3)$ \\
$\mathrm{N} 1-\mathrm{C} 5-\mathrm{C} 4$ & $122.8(3)$ & & & & \\
\hline
\end{tabular}


Dehydrogenative coupling of pyridines by 1 . The reactions of $\mathbf{1}$ with excess 4-substituted pyridines were performed in a glass-tube equipped with a Teflon-valve in appropriate reaction conditions. After the appropriate reaction time, the solution was analyzed by GLC (for pyridine and 4-picoline) and ${ }^{1} \mathrm{H}$ NMR (for 4-methoxypyridine, 4-dimethylaminopyridine, and ethyl isonicotionate) analysis. The results are listed in Table S-5. A typical reaction was carried out as follows (entry 4): $3 \mathrm{~mL}$ of mesitylene solution of 1 (4.0 mM, $0.012 \mathrm{mmol})$ and biphenyl $(48.0 \mathrm{mg}, 0.311 \mathrm{mmol})$ was charged in the reaction flask equipped with Teflon-valve. After a 100 equiv amount of $\gamma$-picoline $(0.118 \mathrm{~mL}, 1.20 \mathrm{mmol})$ was added, the solution was heated at $160{ }^{\circ} \mathrm{C}$ for $19 \mathrm{~h}$. Formation of 4,4'-dimethyl-2,2'-bipyridine was quantitatively analyzed by means of GLC. After the solvent and unreacted $\gamma$-picoline were removed under reduced pressure, the residual solid was analyzed by means of ${ }^{1} \mathrm{H}$ NMR spectroscopy.

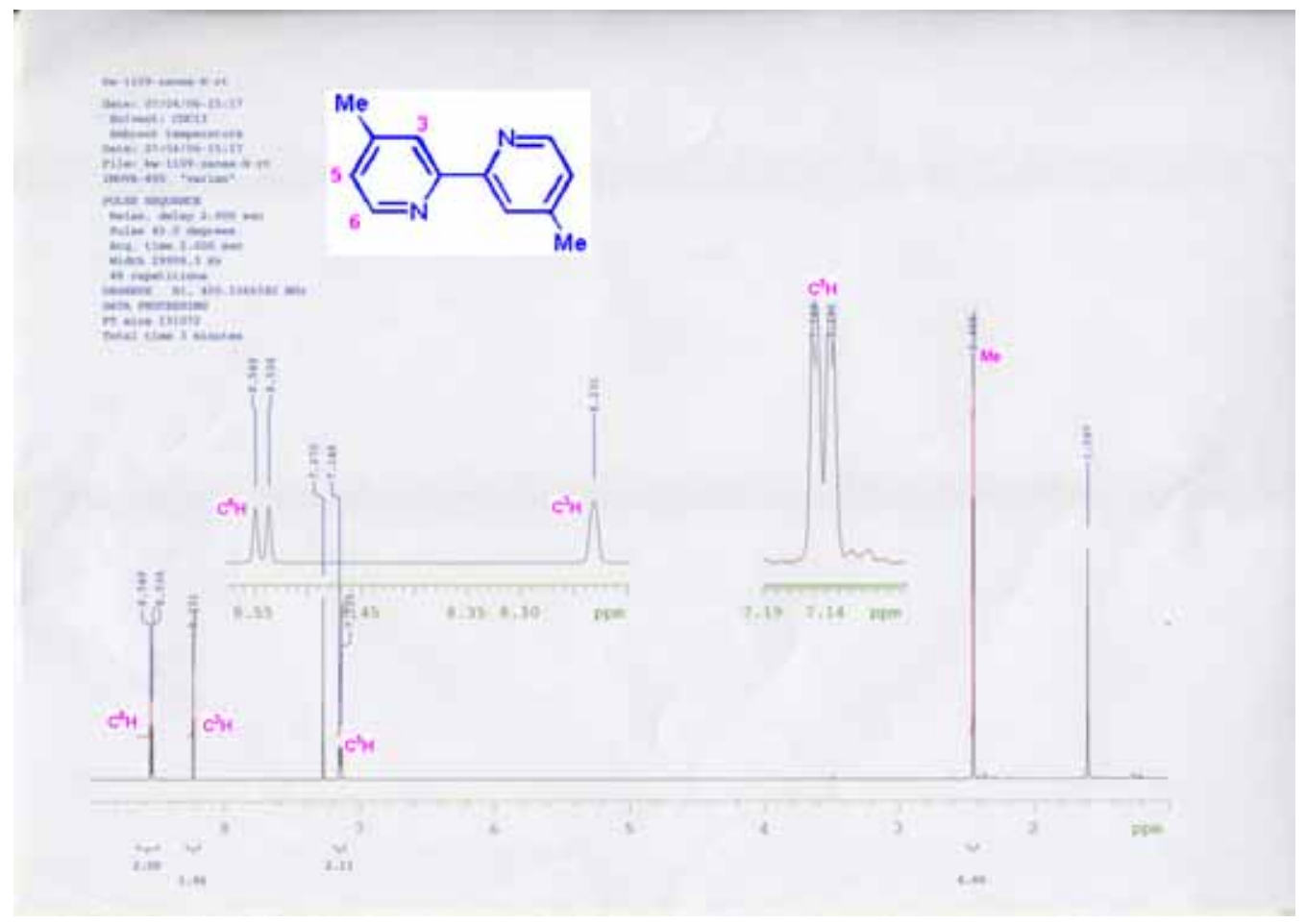

Figure S-1. ${ }^{1} \mathrm{H}$ NMR spectrum of the residual solid obtained by the reaction 5 in Table S-5. 
Table S - 5. Dehydrogenative-coupling of 4-substituted pyridines $\mathrm{C}_{5} \mathrm{H}_{4} \mathrm{RN}$ by $\mathbf{1}^{[\mathrm{a}]}$

\begin{tabular}{ccccccc}
\hline entry & $\mathrm{R}$ & $\mathrm{S} / \mathrm{C}$ & time $[\mathrm{h}]$ & temp. $\left[{ }^{\circ} \mathrm{C}\right]$ & yield $[\%]$ & TON \\
\hline $1^{[\mathrm{b}]}$ & $\mathrm{Me}$ & 100 & 34 & 100 & $0^{[\mathrm{d}]}$ & 0 \\
$2^{[\mathrm{b}]}$ & $\mathrm{Me}$ & 100 & 24 & 120 & $6^{[\mathrm{d}]}$ & 3 \\
3 & $\mathrm{Me}$ & 100 & 6 & 160 & $46^{[\mathrm{d}]}$ & 23 \\
4 & $\mathrm{Me}$ & 100 & 19 & 160 & $53^{[\mathrm{d}]}$ & 26.5 \\
5 & $\mathrm{Me}$ & 500 & 21 & 160 & $21^{\mathrm{d}]}$ & 51.5 \\
6 & $\mathrm{H}$ & 100 & 6 & 160 & $9^{[\mathrm{d}]}$ & 4.5 \\
7 & $\mathrm{OMe}$ & 100 & 6 & 160 & $6^{[\mathrm{e}]}$ & 3 \\
$8^{[\mathrm{c}]}$ & $\mathrm{NMe}_{2}$ & 100 & 6 & 160 & $32^{[\mathrm{e}]}$ & 16 \\
9 & $\mathrm{COOEt}$ & 100 & 6 & 160 & trace $^{[\mathrm{e}]}$ & $-\cdots$ \\
\hline
\end{tabular}

\footnotetext{
${ }^{[a]}$ The reaction was carried out in a glass tube equipped with Teflon-valve. Mesitylene was used as a solvent (ca. 3 $\mathrm{mL}$ ) and the catalyst was loaded as mesitylene solution $(4.0 \mathrm{mM})$. Biphenyl was added to the reaction flask as an internal standard. ${ }^{[\mathrm{b}]}$ Using decane as a solvent and the catalyst was loaded as decane solution. ${ }^{[\mathrm{c}]}$ An internal standard, biphenyl, was not used and the yield was determined by means of ${ }^{1} \mathrm{H}$ NMR by comparing the signal intensities of the product with those of the residual 4-(dimethylamino)pyridine. ${ }^{[\mathrm{d}]}$ The yield was determined by GLC analysis. ${ }^{[\mathrm{e}]}$ The yield was determined by ${ }^{1} \mathrm{H}$ NMR analysis.
}

${ }^{1}$ H NMR data for 4,4-bis(methoxy)-2,2'-bipyridine. (400

$\mathrm{MHz}$, chloroform- $\left.d, 25^{\circ} \mathrm{C}\right): \delta 8.48\left(\mathrm{~d},{ }^{3} \mathrm{~J}(\mathrm{H}, \mathrm{H})=5.6 \mathrm{~Hz}, 2 \mathrm{H}\right.$,

$\left.\mathrm{C}^{6} H\right), 7.99\left(\mathrm{~d},{ }^{4} J(\mathrm{H}, \mathrm{H})=2.8 \mathrm{~Hz}, 2 \mathrm{H}, \mathrm{C}^{3} H\right), 6.85(\mathrm{dd}$,

$\left.{ }^{3} J(\mathrm{H}, \mathrm{H})=5.6,{ }^{4} J(\mathrm{H}, \mathrm{H})=2.8 \mathrm{~Hz}, 2 \mathrm{H}, \mathrm{C}^{5} H\right), 3.96 \mathrm{ppm}(\mathrm{s}, 6 \mathrm{H}$,

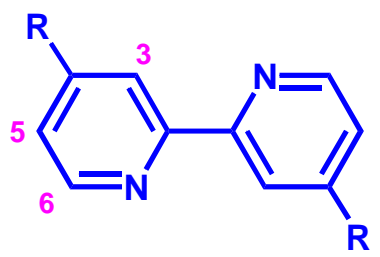

$\mathrm{OMe})$.

${ }^{1}$ H NMR data for 4,4-bis(dimethylamino)-2,2'-bipyridine. (400 MHz, chloroform- $d$, $\left.25^{\circ} \mathrm{C}\right): \delta 8.31\left(\mathrm{~d},{ }^{3} J(\mathrm{H}, \mathrm{H})=5.6 \mathrm{~Hz}, 2 \mathrm{H}, \mathrm{C}^{6} H\right), 7.71\left(\mathrm{~d},{ }^{4} J(\mathrm{H}, \mathrm{H})=2.8 \mathrm{~Hz}, 2 \mathrm{H}, \mathrm{C}^{3} H\right), 6.53$ $\left(\mathrm{dd},{ }^{3} J(\mathrm{H}, \mathrm{H})=5.6,{ }^{4} J(\mathrm{H}, \mathrm{H})=2.8 \mathrm{~Hz}, 2 \mathrm{H}, \mathrm{C}^{5} \mathrm{H}\right), 3.11 \mathrm{ppm}\left(\mathrm{s}, 12 \mathrm{H}, \mathrm{NMe} e_{2}\right)$

${ }^{1}$ H NMR data for 4,4-bis(ethoxycarbonyl)-2,2'-bipyridine. (400 MHz, chloroform- $d$, $\left.25{ }^{\circ} \mathrm{C}\right): \delta 8.95\left(\mathrm{~d},{ }^{4} J(\mathrm{H}, \mathrm{H})=1.6 \mathrm{~Hz}, 2 \mathrm{H}, \mathrm{C}^{3} H\right), 8.87\left(\mathrm{~d},{ }^{3} J(\mathrm{H}, \mathrm{H})=4.8 \mathrm{~Hz}, 2 \mathrm{H}, \mathrm{C}^{6} H\right)$, $7.91\left(\mathrm{dd},{ }^{3} J(\mathrm{H}, \mathrm{H})=4.8,{ }^{4} J(\mathrm{H}, \mathrm{H})=1.6 \mathrm{~Hz}, 2 \mathrm{H}, \mathrm{C}^{5} H\right), 4.46\left(\mathrm{q},{ }^{3} J(\mathrm{H}, \mathrm{H})=7.3 \mathrm{~Hz}, 4 \mathrm{H}\right.$, $\left.\mathrm{CH}_{2} \mathrm{CH}_{3}\right), 1.45 \mathrm{ppm}\left(\mathrm{t},{ }^{3} J(\mathrm{H}, \mathrm{H})=7.3 \mathrm{~Hz}, 6 \mathrm{H}, \mathrm{CH}_{2} \mathrm{CH}_{3}\right)$. 
Dehydrogenative coupling of 4 -substituted pyridines by 2. The reactions of 2 with excess 4-substituted pyridines were performed in a glass tube equipped with a Teflon valve in appropriate reaction conditions. After the appropriate reaction time, the solution was analyzed by GLC (for pyridine and 4-picoline) and ${ }^{1} \mathrm{H}$ NMR (for 4-methoxypyridine, 4-dimethylaminopyridine, and ethyl isonicotionate) analysis. The results are listed in Table S-6. A typical reaction was carried out as follows (entry 5): The $\mu$-dimethylphosphido complex $2(37.1 \mathrm{mg}, 0.061 \mathrm{mmol})$ and biphenyl $(53.7 \mathrm{mg}$, $0.348 \mathrm{mmol}$ ) were dissolved in $20.2 \mathrm{~mL}$ of mesitylene, and a stock-solution of the catalyst precursor $(3.0 \mathrm{mM})$ was formed. A $2.5 \mathrm{~mL}$ amount of the stock-solution was charged in a flask equipped with a Teflon-valve. After 4-methoxy pyridine (76 $\mu \mathrm{L}$, $0.749 \mathrm{mmol}$ ) was added to the flask, the solution was heated at $180{ }^{\circ} \mathrm{C}$ for $20 \mathrm{~h}$. After the solvent and unreacted 4-methoxy pyridine were removed under reduced pressure, the residue was analyzed by means of the ${ }^{1} \mathrm{H}$ NMR spectroscopy. The yield was estimated at $64 \%$ by comparing the signal intensities of the product with those of the internal standard (biphenyl).

Table S - 6. Dehydrogenative-coupling of 4-substituted pyridines $\mathrm{C}_{5} \mathrm{H}_{4} \mathrm{RN}$ by 2. $^{\text {[a] }}$

\begin{tabular}{ccccc}
\hline entry & $\mathrm{R}$ & catalyst & yield [\%] & TON \\
\hline 1 & $\mathrm{Me}$ & $\mathbf{2}$ & $34^{[\mathrm{b}]}$ & 17 \\
2 & $\mathrm{H}$ & $\mathbf{2}$ & $20^{[\mathrm{b}]}$ & 10 \\
3 & $\mathrm{OMe}$ & $\mathbf{2}$ & $64^{[\mathrm{c}]}$ & 32 \\
4 & $\mathrm{NMe}_{2}$ & $\mathbf{2}$ & $8^{[\mathrm{c}]}$ & 4 \\
5 & $\mathrm{COOEt}$ & $\mathbf{2}$ & $20^{[\mathrm{c}]}$ & 10 \\
\hline
\end{tabular}

[a] The reaction was carried out in a glass tube equipped with Teflon-valve at $180^{\circ} \mathrm{C}$ for $20 \mathrm{~h}$. The $\mathrm{S} / \mathrm{C}$ ratio was 100 . Mesitylene was used as a solvent (ca. $3 \mathrm{~mL}$ ) and the catalyst was loaded as mesitylene solution $(3.0 \mathrm{mM})$. Biphenyl was added to the reaction flask as an internal standard. ${ }^{[\mathrm{b}]}$ The yield was determined by GLC analysis. ${ }^{[c]}$ The yield was determined by ${ }^{1} \mathrm{H}$ NMR analysis. 
$(\mathbf{C p} * \mathbf{R u})_{2}\left(\mu-\mathrm{C}_{5} \mathrm{H}_{3}(\mathrm{OMe}) \mathbf{N}\right)_{2}(\boldsymbol{\mu}-\mathrm{H})_{2}$ (3). A 50-mL Schlenk tube was charged with $\mathbf{1}$ (35.8 $\mathrm{mg}, 0.075 \mathrm{mmol}$ ) and THF (3 mL). 4-Methoxy pyridine ( $76 \mu \mathrm{L}, 0.749 \mathrm{mmol}$ ) was added, and the reaction mixture was stirred at $25{ }^{\circ} \mathrm{C}$ for $3 \mathrm{~h}$. Removal of solvent and remaining 4-methoxy pyridine in vacuo afforded a brownish solid. Exclusive formation of 3 was confirmed by the ${ }^{1} \mathrm{H}$ NMR spectra of the residual solid and the yield was estimated at $94 \%$ by the comparison of the intensities of the signals with those of remaining 1. ${ }^{1} \mathrm{H}$ NMR (400 MHz, benzene- $\left.d_{6}, 25^{\circ} \mathrm{C}\right): \delta-9.24(\mathrm{~s}, 2 \mathrm{H}, \mathrm{Ru} H), 1.90(\mathrm{~s}$, $\left.15 \mathrm{H}, \mathrm{C}_{5} M e_{5}\right), 2.05\left(\mathrm{~s}, 15 \mathrm{H}, \mathrm{C}_{5} M e_{5}\right), 3.20(\mathrm{~s}, 6 \mathrm{H}, \mathrm{OMe}), 5.90\left(\mathrm{dd},{ }^{3} J_{\mathrm{H}-\mathrm{H}}=6.4,{ }^{4} J_{\mathrm{H}-\mathrm{H}}=2.8\right.$ $\left.\mathrm{Hz}, 2 \mathrm{H}, \mathrm{C}^{5} H\right), 6.71\left(\mathrm{~d},{ }^{4} J_{\mathrm{H}-\mathrm{H}}=2.8 \mathrm{~Hz}, 2 \mathrm{H}, \mathrm{C}^{3} H\right), 7.89 \mathrm{ppm}\left(\mathrm{d},{ }^{3} J_{\mathrm{H}-\mathrm{H}}=6.4 \mathrm{~Hz}, 2 \mathrm{H}, \mathrm{C}^{6} H\right)$. ${ }^{13} \mathrm{C}$ NMR (100 MHz, benzene- $\left.d_{6}, 25{ }^{\circ} \mathrm{C}\right): \delta 11.3\left(\mathrm{q},{ }^{1} J_{\mathrm{C}-\mathrm{H}}=125 \mathrm{~Hz}, \mathrm{C}_{5} M e_{5}\right), 11.6(\mathrm{q}$, $\left.{ }^{1} J_{\mathrm{C}-\mathrm{H}}=125 \mathrm{~Hz}, \mathrm{C}_{5} M e_{5}\right), 53.8\left(\mathrm{q},{ }^{1} J_{\mathrm{C}-\mathrm{H}}=144 \mathrm{~Hz}\right.$, $\mathrm{OMe}), 80.8\left(\mathrm{~s}_{5} \mathrm{Me}_{5}\right), 96.0\left(\mathrm{~s} C_{5} \mathrm{Me}_{5}\right), 103.1\left(\mathrm{~d},{ }^{1} J_{\mathrm{C}-\mathrm{H}}\right.$ $\left.=163 \mathrm{~Hz}, C^{5}\right), 117.0\left(\mathrm{~d},{ }^{1} J_{\mathrm{C}-\mathrm{H}}=162 \mathrm{~Hz}, C^{3}\right), 148.1(\mathrm{~d}$, $\left.{ }^{1} J_{\mathrm{C}-\mathrm{H}}=174 \mathrm{~Hz}, C^{6}\right), 160.2\left(\mathrm{~s}, C^{4}\right), 204.4 \mathrm{ppm}\left(\mathrm{s}, C^{2}\right)$. Due to instability to the air, elemental analysis of $\mathbf{3}$ cannot be performed.

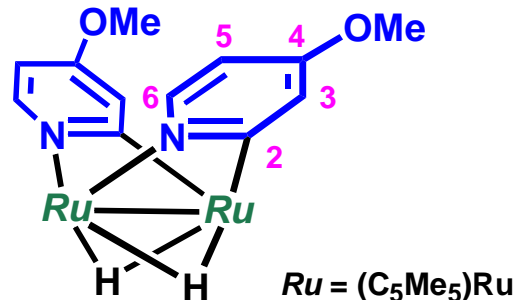

3

$\left(\mathrm{Cp}^{*} \mathbf{R u}\right)_{2}(\boldsymbol{\mu}-\mathbf{P M e})\left(\boldsymbol{\mu}-\mathrm{C}_{5} \mathbf{H}_{4} \mathbf{N}\right)(\boldsymbol{\mu}-\mathbf{H})(\mathbf{H})($ (4). A 50-mL Schlenk tube was charged with THF (10 mL), 2 (106.3 mg, $0.174 \mathrm{mmol})$, and 10 equiv amount of pyridine (140 $\mu \mathrm{L}$, $1.740 \mathrm{mmol})$. The reaction mixture was heated at $60{ }^{\circ} \mathrm{C}$ for $24 \mathrm{~h}$. The color of the solution turned from red to orange. The solvent and remaining pyridine were removed under reduced pressure. The residual solid was then purified by the use of column chromatography on alumina (Merck, Art. No. 1097) with pentane and toluene (1/1). The first orange band including 4 was collected, and the solvent was removed under reduced pressure. A $50.9 \mathrm{mg}$ amount of 4 was obtained as an orange solid (0.0829 mmol, $48 \%$ ). 
${ }^{1} \mathrm{H}$ NMR $\left(400 \mathrm{MHz}, \mathrm{THF}-d_{8}, 25{ }^{\circ} \mathrm{C}\right): \delta-10.92(\mathrm{~d}$, $\left.J_{\mathrm{P}-\mathrm{H}}=21.6 \mathrm{~Hz}, 1 \mathrm{H}, \mathrm{Ru} H \mathrm{Ru}\right),-9.34\left(\mathrm{~d}, J_{\mathrm{P}-\mathrm{H}}=10.8\right.$ $\mathrm{Hz}, 1 \mathrm{H}, \mathrm{Ru} H), 1.13\left(\mathrm{~d}, J_{\mathrm{P}-\mathrm{H}}=9.6 \mathrm{~Hz}, 3 \mathrm{H}, \mathrm{P} M e_{2}\right)$, $1.59\left(\mathrm{~d}, J_{\mathrm{P}-\mathrm{H}}=10.0 \mathrm{~Hz}, 3 \mathrm{H}, \mathrm{P} M e_{2}\right), 1.89\left(\mathrm{~d}, J_{\mathrm{P}-\mathrm{H}}=\right.$ $\left.1.6 \mathrm{~Hz}, 15 \mathrm{H}, \mathrm{C}_{5} M e_{5}\right), 1.99\left(\mathrm{~s}, 15 \mathrm{H}, \mathrm{C}_{5} M e_{5}\right), 6.05$

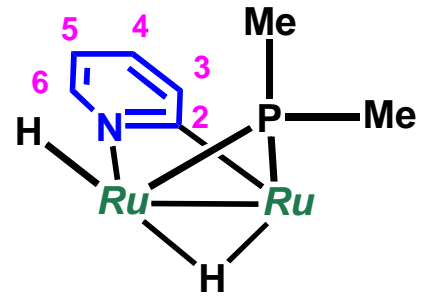

$4 \quad \mathrm{Ru}=\left(\mathrm{C}_{5} \mathrm{Me}_{5}\right) \mathrm{Ru}$ $\left(\mathrm{dd}, J_{\mathrm{H}-\mathrm{H}}=7.2,5.6 \mathrm{~Hz}, 1 \mathrm{H}, \mathrm{C}^{5} H\right), 6.41\left(\mathrm{dd}, J_{\mathrm{H}-\mathrm{H}}=8.0,7.2 \mathrm{~Hz}, 1 \mathrm{H}, \mathrm{C}^{4} H\right), 6.57\left(\mathrm{~d}, J_{\mathrm{H}-\mathrm{H}}=\right.$ $\left.8.0 \mathrm{~Hz}, 1 \mathrm{H}, \mathrm{C}^{3} H\right), 7.42 \mathrm{ppm}\left(\mathrm{d}, J_{\mathrm{H}-\mathrm{H}}=7.2 \mathrm{~Hz}, 1 \mathrm{H}, \mathrm{C}^{6} H\right) .{ }^{13} \mathrm{C}$ NMR $\left(100 \mathrm{MHz}, \mathrm{THF}-d_{8}\right.$, $\left.25^{\circ} \mathrm{C}\right): \delta 11.9\left(\mathrm{q}, J_{\mathrm{C}-\mathrm{H}}=126 \mathrm{~Hz}, \mathrm{C}_{5} M e_{5}\right), 12.3\left(\mathrm{q}, J_{\mathrm{C}-\mathrm{H}}=125 \mathrm{~Hz}, \mathrm{C}_{5} M e_{5}\right), 20.8\left(\mathrm{qd}, J_{\mathrm{C}-\mathrm{H}}\right.$ $\left.=124 \mathrm{~Hz}, J_{\mathrm{C}-\mathrm{P}}=18 \mathrm{~Hz}, \mathrm{P} M e_{2}\right), 21.1\left(\mathrm{qd}, J_{\mathrm{C}-\mathrm{H}}=126 \mathrm{~Hz}, J_{\mathrm{C}-\mathrm{P}}=15 \mathrm{~Hz}, \mathrm{P} M e_{2}\right), 87.0(\mathrm{~s}$ $\left.C_{5} \mathrm{Me}_{5}\right), 96.2\left(\mathrm{~s} C_{5} \mathrm{Me}_{5}\right), 115.3\left(\mathrm{~d}, J_{\mathrm{C}-\mathrm{H}}=163 \mathrm{~Hz}, C^{5}\right), 125.7\left(\mathrm{~d}, J_{\mathrm{C}-\mathrm{H}}=161 \mathrm{~Hz}, C^{4}\right)$, $132.0\left(\mathrm{~d}, J_{\mathrm{C}-\mathrm{H}}=160 \mathrm{~Hz}, C^{3}\right), 149.2\left(\mathrm{~d}, J_{\mathrm{C}-\mathrm{H}}=171 \mathrm{~Hz}, C^{6}\right), 192.4 \mathrm{ppm}\left(\mathrm{s}, C^{2}\right) .{ }^{31} \mathrm{P}\left\{{ }^{1} \mathrm{H}\right\}$ NMR (162 MHz, THF- $\left.d_{8}, 25^{\circ} \mathrm{C}\right): \delta 151.1 \mathrm{ppm}$. Anal. Calcd for $\mathrm{C}_{27} \mathrm{H}_{42} \mathrm{~N}_{1} \mathrm{P}_{1} \mathrm{Ru}_{2}: \mathrm{C}$, 52.84; H, 6.90; N, 2.28. Found: C, 52.58; H, 6.61; N, 2.03. Preliminary result of an X-ray diffraction study for $\mathbf{4}$ showed that one of the hydrido ligands is terminally bonded to the ruthenium atom (Figure S-2). But, due to the disorder arising from the bridging pyridyl group (N1 and C5), the position of the terminal hydrido ligand could not be determined. We tentatively assigned the position of the terminal hydrido ligand at the ruthenium center, to which the nitrogen atom of the $\mu$-pyridyl ligand is bonded as shown in Figure S-2.

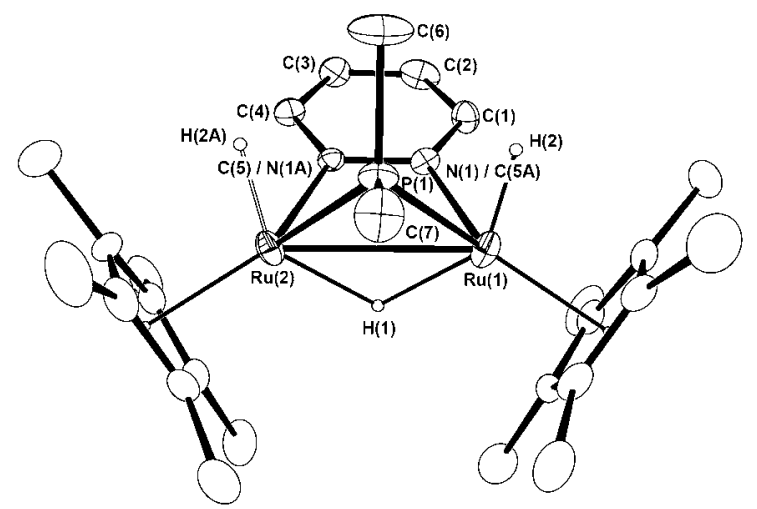

Figure S-2. Molecular structure and labeling scheme of 4 with thermal ellipsoids at the $30 \%$ level of probability. 


\section{$\left(C^{*} \mathrm{Ru}\right)_{2}\left(\mu-\mathrm{PMe}_{2}\right)\left(\mu-\eta^{2}: \eta^{2}-2,2^{\prime}-\mathrm{bpy}\right)(\mu-\mathrm{H}) \quad$ (5). A}

20-mL glass tube equipped with J-Young valve was charged with $2 \quad(56.2 \quad \mathrm{mg}, \quad 0.0917 \mathrm{mmol})$, 2,2'-bipyridine (292.4 $\mathrm{mg}, 1.872 \mathrm{mmol})$ and $\mathrm{THF}$ $(10 \mathrm{~mL})$. The reaction mixture was heated at $80{ }^{\circ} \mathrm{C}$ for $24 \mathrm{~h}$. After the solvent was removed under

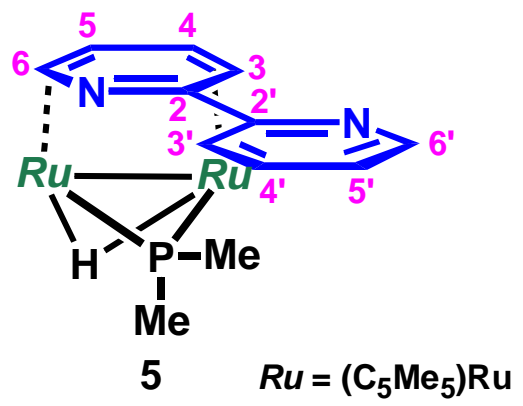
reduced pressure, the residual solid was purified by the use of column chromatography on neutral alumina (Merck, Art. No. 1097) with THF. The third purple band including 4 was collected, and the fraction was dried in vacuo. A $26.6 \mathrm{mg}$ amount of $\mathbf{4}$ was obtained as a red solid (0.0385 mmol, $42 \%) .{ }^{1} \mathrm{H}$ NMR (400 MHz, THF- $\left.d_{8}, 25{ }^{\circ} \mathrm{C}\right): \delta-17.52(\mathrm{~d}$, $\left.{ }^{2} J(\mathrm{P}, \mathrm{H})=36.4 \mathrm{~Hz}, 1 \mathrm{H}, \mathrm{Ru} H\right), 0.55\left(\mathrm{~d},{ }^{2} J_{\mathrm{P}-\mathrm{H}}=10.0 \mathrm{~Hz}, 3 \mathrm{H}, \mathrm{P} M e_{2}\right), 1.06\left(\mathrm{~d},{ }^{2} J_{\mathrm{P}-\mathrm{H}}=9.6\right.$ $\left.\mathrm{Hz}, 3 \mathrm{H}, \mathrm{P} M e_{2}\right), 1.82\left(\mathrm{~d},{ }^{4} J_{\mathrm{P}-\mathrm{H}}=0.8 \mathrm{~Hz}, 15 \mathrm{H}, \mathrm{C}_{5} M e_{5}\right), 1.83\left(\mathrm{~d},{ }^{4} J_{\mathrm{P}-\mathrm{H}}=0.8 \mathrm{~Hz}, 15 \mathrm{H}\right.$, $\left.\mathrm{C}_{5} M e_{5}\right), 2.35\left(\mathrm{ddd},{ }^{3} J_{\mathrm{H}-\mathrm{H}}=5.6,{ }^{3} J_{\mathrm{H}-\mathrm{H}}=4.8,{ }^{4} J_{\mathrm{H}-\mathrm{H}}=1.2 \mathrm{~Hz}, 1 \mathrm{H}, \mathrm{C}^{5} H\right), 2.60\left(\mathrm{ddd},{ }^{3} J_{\mathrm{H}-\mathrm{H}}=\right.$ $\left.7.2,{ }^{3} J_{\mathrm{H}-\mathrm{H}}=5.6,{ }^{4} J_{\mathrm{H}-\mathrm{H}}=1.6 \mathrm{~Hz}, 1 \mathrm{H}, \mathrm{C}^{4} H\right), 3.74\left(\mathrm{dd},{ }^{3} J_{\mathrm{H}-\mathrm{H}}=7.2,{ }^{4} J_{\mathrm{H}-\mathrm{H}}=1.2 \mathrm{~Hz}, 1 \mathrm{H}\right.$, $\left.\mathrm{C}^{3} H\right), 4.34\left(\mathrm{dd},{ }^{3} J_{\mathrm{H}-\mathrm{H}}=4.8,{ }^{4} J_{\mathrm{H}-\mathrm{H}}=1.6 \mathrm{~Hz}, 1 \mathrm{H}, \mathrm{C}^{6} H\right), 7.02\left(\mathrm{ddd},{ }^{3} J_{\mathrm{H}-\mathrm{H}}=7.2,{ }^{3} J_{\mathrm{H}-\mathrm{H}}=4.8\right.$, $\left.{ }^{4} J_{\mathrm{H}-\mathrm{H}}=0.8 \mathrm{~Hz}, 1 \mathrm{H}, \mathrm{C}^{5} H\right), 7.52\left(\mathrm{ddd},{ }^{3} J_{\mathrm{H}-\mathrm{H}}=8.0,{ }^{3} J_{\mathrm{H}-\mathrm{H}}=7.2,{ }^{4} J_{\mathrm{H}-\mathrm{H}}=1.6 \mathrm{~Hz}, 1 \mathrm{H}, \mathrm{C}^{4} H\right)$, $7.99\left(\mathrm{dd},{ }^{3} J_{\mathrm{H}-\mathrm{H}}=8.0,{ }^{4} J_{\mathrm{H}-\mathrm{H}}=0.8 \mathrm{~Hz}, 1 \mathrm{H}, \mathrm{C}^{3} \mathrm{H}\right), 8.41 \mathrm{ppm}\left(\mathrm{dd},{ }^{3} J_{\mathrm{H}-\mathrm{H}}=4.8,{ }^{4} J_{\mathrm{H}-\mathrm{H}}=1.6\right.$ $\left.\mathrm{Hz}, 1 \mathrm{H}, \mathrm{C}^{6} H\right) .{ }^{13} \mathrm{C}$ NMR $\left(100 \mathrm{MHz}, \mathrm{THF}-d_{8}, 25{ }^{\circ} \mathrm{C}\right): \delta 6.5\left(\mathrm{qd},{ }^{1} J_{\mathrm{C}-\mathrm{H}}=128 \mathrm{~Hz},{ }^{1} J_{\mathrm{C}-\mathrm{P}}=\right.$ $\left.6 \mathrm{~Hz}, \mathrm{P} M e_{2}\right), 10.8_{8}\left(\mathrm{q},{ }^{1} J_{\mathrm{C}-\mathrm{H}}=126 \mathrm{~Hz}, \mathrm{C}_{5} M e_{5}\right), 10.9_{2}\left(\mathrm{q},{ }^{1} J_{\mathrm{C}-\mathrm{H}}=126 \mathrm{~Hz}, \mathrm{C}_{5} M e_{5}\right), 25.4$ $\left(\mathrm{qd},{ }^{1} J_{\mathrm{C}-\mathrm{H}}=126 \mathrm{~Hz},{ }^{1} J_{\mathrm{C}-\mathrm{P}}=19 \mathrm{~Hz}, \mathrm{P} M e_{2}\right), 37.2\left(\mathrm{~d},{ }^{1} J_{\mathrm{C}-\mathrm{H}}=160 \mathrm{~Hz}, C^{3}\right), 44.8\left(\mathrm{~d},{ }^{1} J_{\mathrm{C}-\mathrm{H}}=\right.$ $\left.159 \mathrm{~Hz}, C^{5}\right), 49.4\left(\mathrm{~d},{ }^{1} J_{\mathrm{C}-\mathrm{H}}=161 \mathrm{~Hz}, C^{4}\right), 68.3\left(\mathrm{~d},{ }^{1} J_{\mathrm{C}-\mathrm{H}}=171 \mathrm{~Hz}, C^{6}\right), 93.9\left(\mathrm{~s}_{5} \mathrm{Me}_{5}\right)$, $94.2\left(\mathrm{~s} C_{5} \mathrm{Me}_{5}\right), 120.0\left(\mathrm{~d},{ }^{1} J_{\mathrm{C}-\mathrm{H}}=165 \mathrm{~Hz}, C^{3}\right), 122.1\left(\mathrm{~d},{ }^{1} J_{\mathrm{C}-\mathrm{H}}=163 \mathrm{~Hz}, C^{5}\right), 135.3(\mathrm{~d}$, $\left.{ }^{1} J_{\mathrm{C}-\mathrm{H}}=160 \mathrm{~Hz}, C^{4}\right), 148.5\left(\mathrm{~d},{ }^{1} J_{\mathrm{C}-\mathrm{H}}=175 \mathrm{~Hz}, C^{6}\right), 158.6\left(\mathrm{~s}, C^{2}\right), 159.5 \mathrm{ppm}\left(\mathrm{s}, C^{2}\right)$. ${ }^{31} \mathrm{P}\left\{{ }^{1} \mathrm{H}\right\}$ NMR $\left(162 \mathrm{MHz}, \mathrm{THF}-d_{8}, 25{ }^{\circ} \mathrm{C}\right): \delta 124.5 \mathrm{ppm}$. Elemental analysis was performed on the diphenylphosphido analogue 5' as described below. 


\begin{tabular}{ccc}
\hline H-H COSY & HMQC & HMBC \\
\hline$\delta 2.35-\delta 2.60,4.34$ & $\delta_{\mathrm{H}} 2.35-\delta_{\mathrm{C}} 44.8$ & $\delta_{\mathrm{H}} 2.35-\delta_{\mathrm{C}} 37.2,49.4,68.3$ \\
$\delta 2.60-\delta 3.74,4.34$ & $\delta_{\mathrm{H}} 2.60-\delta_{\mathrm{C}} 49.4$ & $\delta_{\mathrm{H}} 2.60-\delta_{\mathrm{C}} 37.2,44.8,68.3,159.5$ \\
$\delta 7.02-\delta 7.52,8.41$ & $\delta_{\mathrm{H}} 3.74-\delta_{\mathrm{C}} 37.2$ & $\delta_{\mathrm{H}} 3.74-\delta_{\mathrm{C}} 44.8$ \\
$\delta 7.52-\delta 7.99$ & $\delta_{\mathrm{H}} 4.34-\delta_{\mathrm{C}} 68.3$ & $\delta_{\mathrm{H}} 4.34-\delta_{\mathrm{C}} 44.8,49.4,159.5$ \\
& $\delta_{\mathrm{H}} 7.02-\delta_{\mathrm{C}} 122.1$ & $\delta_{\mathrm{H}} 7.02-\delta_{\mathrm{C}} 120.0,148.5$ \\
& $\delta_{\mathrm{H}} 7.52-\delta_{\mathrm{C}} 135.3$ & $\delta_{\mathrm{H}} 7.52-\delta_{\mathrm{C}} 148.5,158.6$ \\
& $\delta_{\mathrm{H}} 7.99-\delta_{\mathrm{C}} 120.0$ & $\delta_{\mathrm{H}} 7.99-\delta_{\mathrm{C}} 122.1$ \\
& $\delta_{\mathrm{H}} 8.41-\delta_{\mathrm{C}} 148.5$ & $\delta_{\mathrm{H}} 8.41-\delta_{\mathrm{C}} 122.1,135.3$ \\
\hline
\end{tabular}

$\left(\mathbf{C P} \mathbf{P}^{*} \mathbf{R u}\right)_{2}\left(\boldsymbol{\mu}-\mathbf{P P h} \mathbf{h}_{2}\right)\left(\boldsymbol{\mu}-\eta^{2}: \eta^{2}-2,2^{\prime}-\mathbf{b p y}\right)(\boldsymbol{\mu}-\mathbf{H})$ (5'). A $20-\mathrm{mL}$ glass tube equipped with J-Young valve was charged with 2' (34.2 mg, $0.0464 \mathrm{mmol}), 2$,' '-bipyridine (153.0 mg, $0.980 \mathrm{mmol}, 21$ equiv), and THF ( $5 \mathrm{~mL})$. The reaction mixture was heated at $40{ }^{\circ} \mathrm{C}$ for $24 \mathrm{~h}$. After the solvent was removed under reduced pressure, the residual solid was purified by the use of column chromatography on neutral alumina (Merck, Art. No. 1097) with pentane/tetrahydrofuran (1:1). The third red band including 4' was collected, and the fraction was dried in vacuo. A $16.0 \mathrm{mg}$ amount of 4' was obtained as a red solid (0.0196 mmol, $42 \%) .{ }^{1} \mathrm{H}$ NMR (400 MHz, THF- $\left.d_{8}, 25{ }^{\circ} \mathrm{C}\right): \delta-17.19(\mathrm{~d}$, $\left.{ }^{2} J_{\mathrm{H}-\mathrm{P}}=39.2 \mathrm{~Hz}, 1 \mathrm{H}, \mathrm{Ru} H\right), 1.57\left(\mathrm{~d},{ }^{4} J_{\mathrm{H}-\mathrm{P}}=0.8 \mathrm{~Hz}, 15 \mathrm{H}, \mathrm{C}_{5} M e_{5}\right), 1.66\left(\mathrm{~d},{ }^{4} J_{\mathrm{H}-\mathrm{P}}=0.8 \mathrm{~Hz}\right.$, $\left.15 \mathrm{H}, \mathrm{C}_{5} M e_{5}\right), 2.36\left(\mathrm{ddd},{ }^{3} J_{\mathrm{H}-\mathrm{H}}=5.6,{ }^{3} J_{\mathrm{H}-\mathrm{H}}=4.8,{ }^{4} J_{\mathrm{H}-\mathrm{H}}=1.2 \mathrm{~Hz}, 1 \mathrm{H}, \mathrm{C}^{5} H\right), 2.67(\mathrm{ddd}$, $\left.{ }^{3} J_{\mathrm{H}-\mathrm{H}}=7.2,{ }^{3} J_{\mathrm{H}-\mathrm{H}}=5.6,{ }^{4} J_{\mathrm{H}-\mathrm{H}}=2.0 \mathrm{~Hz}, 1 \mathrm{H}, \mathrm{C}^{4} H\right), 3.86\left(\mathrm{dd},{ }^{3} J_{\mathrm{H}-\mathrm{H}}=7.2,{ }^{4} J_{\mathrm{H}-\mathrm{H}}=1.2 \mathrm{~Hz}\right.$, $\left.1 \mathrm{H}, \mathrm{C}^{3} H\right), 4.42\left(\mathrm{dd},{ }^{3} J_{\mathrm{H}-\mathrm{H}}=4.8,{ }^{4} J_{\mathrm{H}-\mathrm{H}}=2.0 \mathrm{~Hz}, 1 \mathrm{H}, \mathrm{C}^{6} H\right), 6.07\left(\mathrm{dd},{ }^{3} J_{\mathrm{H}-\mathrm{H}}=7.6,{ }^{3} J_{\mathrm{H}-\mathrm{H}}=\right.$ $7.6 \mathrm{~Hz}, 1 \mathrm{H}$, syn-Ph), $6.25\left(\mathrm{br}, 2 \mathrm{H}\right.$, anti-Ph), $6.62\left(\mathrm{dd},{ }^{3} J_{\mathrm{H}-\mathrm{H}}=8.8,{ }^{3} J_{\mathrm{H}-\mathrm{H}}=7.6 \mathrm{~Hz}, 1 \mathrm{H}\right.$, syn-Ph), $6.65\left(\mathrm{ddd},{ }^{3} J_{\mathrm{H}-\mathrm{H}}=7.6,{ }^{3} J_{\mathrm{H}-\mathrm{H}}=7.6,{ }^{4} J_{\mathrm{H}-\mathrm{H}}=1.6 \mathrm{~Hz}, 1 \mathrm{H}\right.$, syn-Ph), $6.74\left(\mathrm{dd},{ }^{3} J_{\mathrm{H}-\mathrm{H}}\right.$ $\left.=4.8,{ }^{3} J_{\mathrm{H}-\mathrm{H}}=4.4 \mathrm{~Hz}, 1 \mathrm{H}, \mathrm{C}^{5} H\right), 6.75\left(\mathrm{t},{ }^{3} J_{\mathrm{H}-\mathrm{H}}=4.8 \mathrm{~Hz}, 1 \mathrm{H}\right.$, anti-Ph), $6.77(\mathrm{br}, 2 \mathrm{H}$, anti-Ph), $6.86\left(\mathrm{ddd},{ }^{3} J_{\mathrm{H}-\mathrm{H}}=7.6,{ }^{3} J_{\mathrm{H}-\mathrm{H}}=7.6,{ }^{4} J_{\mathrm{H}-\mathrm{H}}=1.6 \mathrm{~Hz}, 1 \mathrm{H}\right.$, syn-Ph $), 6.91(\mathrm{~m}, 2 \mathrm{H}$, $\left.\mathrm{C}^{4^{\prime}} H+\mathrm{C}^{3} H\right), 7.28\left(\mathrm{dd},{ }^{3} J_{\mathrm{H}-\mathrm{H}}=8.8,{ }^{3} J_{\mathrm{H}-\mathrm{H}}=7.6 \mathrm{~Hz}, 1 \mathrm{H}, \mathrm{syn}-P h\right), 8.13 \mathrm{ppm}\left(\mathrm{dd},{ }^{3} J_{\mathrm{H}-\mathrm{H}}=\right.$ $\left.4.8,{ }^{4} J_{\mathrm{H}-\mathrm{H}}=1.2 \mathrm{~Hz}, 1 \mathrm{H}, \mathrm{C}^{6^{\prime}} H\right) \cdot{ }^{13} \mathrm{C}$ NMR $\left(100 \mathrm{MHz}, \mathrm{THF}-d_{8}, 25^{\circ} \mathrm{C}\right): \delta 10.2\left(\mathrm{q},{ }^{1} J_{\mathrm{C}-\mathrm{H}}=\right.$ $\left.126 \mathrm{~Hz}, \mathrm{C}_{5} M e_{5}\right), 10.4\left(\mathrm{q},{ }^{1} J_{\mathrm{C}-\mathrm{H}}=127 \mathrm{~Hz}, \mathrm{C}_{5} M e_{5}\right), 37.0\left(\mathrm{~d},{ }^{1} J_{\mathrm{C}-\mathrm{H}}=159 \mathrm{~Hz}, C^{3}\right), 43.6(\mathrm{~d}$, $\left.{ }^{1} J_{\mathrm{C}-\mathrm{H}}=159 \mathrm{~Hz}, C^{5}\right), 48.2\left(\mathrm{~d},{ }^{1} J_{\mathrm{C}-\mathrm{H}}=164 \mathrm{~Hz}, C^{4}\right), 66.4\left(\mathrm{~d},{ }^{1} J_{\mathrm{C}-\mathrm{H}}=178 \mathrm{~Hz}, C^{6}\right), 95.0(\mathrm{~s}$ 
$\left.C_{5} \mathrm{Me}_{5}\right), 95.3\left(\mathrm{~s}_{5} \mathrm{Me}_{5}\right), 119.2\left(\mathrm{~d},{ }^{1} J_{\mathrm{C}-\mathrm{H}}=167 \mathrm{~Hz}\right.$, $\left.C^{3}\right), 122.0\left(\mathrm{~d},{ }^{1} J_{\mathrm{C}-\mathrm{H}}=161 \mathrm{~Hz}, C^{5}\right), 125.2\left(\mathrm{dd},{ }^{1} J_{\mathrm{C}-\mathrm{H}}\right.$ $=154 \mathrm{~Hz}, J_{\mathrm{C}-\mathrm{P}}=12 \mathrm{~Hz}$, syn-Ph), $125.3\left(\mathrm{dd},{ }^{1} J_{\mathrm{C}-\mathrm{H}}=\right.$ $158 \mathrm{~Hz}, J_{\mathrm{C}-\mathrm{P}}=12 \mathrm{~Hz}$, syn-Ph $), 126.3\left(\mathrm{~d},{ }^{1} J(\mathrm{C}, \mathrm{H})=\right.$ $158 \mathrm{~Hz}$, anti-Ph), $126.4\left(\mathrm{dd},{ }^{1} J(\mathrm{C}, \mathrm{H})=158 \mathrm{~Hz}, J_{\mathrm{C}-\mathrm{P}}\right.$ $=12 \mathrm{~Hz}$, anti-Ph), $127.8\left(\mathrm{dd},{ }^{1} J_{\mathrm{C}-\mathrm{H}}=157 \mathrm{~Hz}, J_{\mathrm{C}-\mathrm{P}}=\right.$

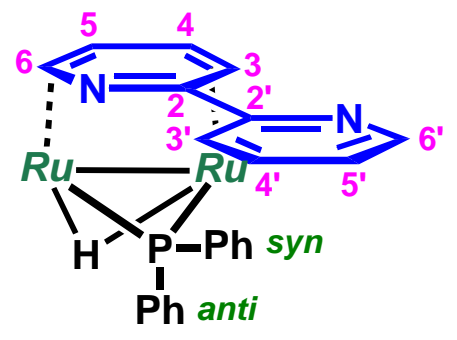

$5^{\prime} \quad R u=\left(\mathrm{C}_{5} \mathrm{Me}_{5}\right) \mathrm{Ru}$

$2 \mathrm{~Hz}$, syn-Ph), 134.2 (d, $\left.{ }^{1} J_{\mathrm{C}-\mathrm{P}}=27 \mathrm{~Hz}, \operatorname{syn}-P h\right), 134.7\left(\mathrm{~d},{ }^{1} J_{\mathrm{C}-\mathrm{H}}=161 \mathrm{~Hz}, C^{4}\right), 134.8$ (br, anti-Ph), $137.3\left(\mathrm{dd},{ }^{1} J_{\mathrm{C}-\mathrm{H}}=160 \mathrm{~Hz}, J_{\mathrm{C}-\mathrm{P}}=17 \mathrm{~Hz}\right.$, syn-Ph), $137.4\left(\mathrm{dd},{ }^{1} J_{\mathrm{C}-\mathrm{H}}=161 \mathrm{~Hz}\right.$, $J_{\mathrm{C}-\mathrm{P}}=19 \mathrm{~Hz}$, syn-Ph), $147.3\left(\mathrm{~d},{ }^{1} J_{\mathrm{C}-\mathrm{H}}=174 \mathrm{~Hz}, C^{6}\right), 151.4\left(\mathrm{~d},{ }^{1} J_{\mathrm{C}-\mathrm{P}}=25 \mathrm{~Hz}\right.$, anti-Ph), $157.5\left(\mathrm{~s}, C^{2}\right), 160.3 \mathrm{ppm}\left(\mathrm{s}, C^{2}\right) .{ }^{31} \mathrm{P}\left\{{ }^{1} \mathrm{H}\right\}$ NMR (162 MHz, THF- $\left.d_{8}, 25{ }^{\circ} \mathrm{C}\right): \delta 166.2$ ppm. Anal. Calcd for $\mathrm{C}_{42} \mathrm{H}_{57} \mathrm{~N}_{2} \mathrm{P}_{1} \mathrm{Ru}_{2}$ : C, 61.90; H, 6.06; N, 3.44. Found: C, 61.78; H, $6.46 ; \mathrm{N}, 3.59$.

\begin{tabular}{ccc}
\hline H-H COSY & HMQC & HMBC \\
\hline$\delta 2.36-\delta 2.67,3.86,4.42$ & $\delta_{\mathrm{H}} 2.36-\delta_{\mathrm{C}} 43.6$ & $\delta_{\mathrm{H}} 2.36-\delta_{\mathrm{C}} 37.0,48.2,66.4$ \\
$\delta 2.67-\delta 3.86,4.42$ & $\delta_{\mathrm{H}} 2.67-\delta_{\mathrm{C}} 48.2$ & $\delta_{\mathrm{H}} 2.67-\delta_{\mathrm{C}} 37.0,43.6,66.4,160.3$ \\
$\delta 6.07-\delta 6.62,6.67,6.86$ & $\delta_{\mathrm{H}} 3.86-\delta_{\mathrm{C}} 37.0$ & $\delta_{\mathrm{H}} 3.86-\delta_{\mathrm{C}} 43.6,157.5$ \\
$\delta 6.62-\delta 6.67,7.28$ & $\delta_{\mathrm{H}} 4.72-\delta_{\mathrm{C}} 66.4$ & $\delta_{\mathrm{H}} 4.72-\delta_{\mathrm{C}} 43.6,48.2,160.3$ \\
$\delta 6.67-\delta 6.86,7.28$ & $\delta_{\mathrm{H}} 6.07-\delta_{\mathrm{C}} 125.3$ & $\delta_{\mathrm{H}} 6.07-\delta_{\mathrm{C}} 125.2,134.2$ \\
$\delta 6.74-\delta 6.91,8.13$ & $\delta_{\mathrm{H}} 6.62-\delta_{\mathrm{C}} 137.3$ & $\delta_{\mathrm{H}} 6.62-\delta_{\mathrm{C}} 127.8,137.4$ \\
$\delta 6.86-\delta 7.28$ & $\delta_{\mathrm{H}} 6.65-\delta_{\mathrm{C}} 127.8$ & $\delta_{\mathrm{H}} 6.65-\delta_{\mathrm{C}} 137.3$ \\
$\delta 6.91-\delta 8.13$ & $\delta_{\mathrm{H}} 6.74-\delta_{\mathrm{C}} 122.0$ & $\delta_{\mathrm{H}} 6.74-\delta_{\mathrm{C}} 119.2,147.3,157.5$ \\
& $\delta_{\mathrm{H}} 6.75-\delta_{\mathrm{C}} 126.3$ & $\delta_{\mathrm{H}} 6.86-\delta_{\mathrm{C}} 125.3,134.2$ \\
& $\delta_{\mathrm{H}} 6.77-\delta_{\mathrm{C}} 126.4$ & $\delta_{\mathrm{H}} 6.91-\delta_{\mathrm{C}} 122.0,147.3,157.5,160.3$ \\
& $\delta_{\mathrm{H}} 6.86-\delta_{\mathrm{C}} 125.2$ & $\delta_{\mathrm{H}} 7.28-\delta_{\mathrm{C}} 127.8,137.3$ \\
& $\delta_{\mathrm{H}} 6.91-\delta_{\mathrm{C}} 119.2,134.7$ & $\delta_{\mathrm{H}} 8.13-\delta_{\mathrm{C}} 122.0,134.7,157.5$ \\
\hline
\end{tabular}

Spectral data of $\left(\mathbf{C} \mathbf{p}^{*} \mathbf{R u}\right)_{2}(\boldsymbol{\mu}-\mathbf{P M e})\left(\boldsymbol{\mu}-\boldsymbol{\eta}^{2}: \boldsymbol{\eta}^{2}-\mathbf{C}_{6} \mathbf{H}_{\mathbf{6}}\right)(\boldsymbol{\mu}-\mathbf{H})(\mathbf{2 a}) .{ }^{1} \mathrm{H}$ NMR (300 MHz, benzene- $\left.d_{6}, 25^{\circ} \mathrm{C}\right): \delta-16.98\left(\mathrm{~d},{ }^{2} J_{\mathrm{P}-\mathrm{H}}=38.3 \mathrm{~Hz}, 1 \mathrm{H}, \mathrm{Ru} H\right), 1.12\left(\mathrm{~d},{ }^{2} J_{\mathrm{P}-\mathrm{H}}=9.2 \mathrm{~Hz}, 3 \mathrm{H}\right.$, $\mathrm{PMe}), 1.21\left(\mathrm{~d},{ }^{2} J_{\mathrm{P}-\mathrm{H}}=9.5 \mathrm{~Hz}, 3 \mathrm{H}, \mathrm{P} M e\right), 1.72\left(\mathrm{~s}, 30 \mathrm{H}, \mathrm{C}_{5} M e_{5}\right), 2.67\left(\mathrm{~m}, 2 \mathrm{H}, \mathrm{C}_{6} H_{6}\right), 2.75$ $\left(\mathrm{m}, 2 \mathrm{H}, \mathrm{C}_{6} H_{6}\right), 6.13 \mathrm{ppm}\left(\mathrm{m}, 2 \mathrm{H}, \mathrm{C}_{6} H_{6}\right) .{ }^{13} \mathrm{C}$ NMR $\left(75.4 \mathrm{MHz}\right.$, benzene- $\left.d_{6}, 25{ }^{\circ} \mathrm{C}\right): \delta$ $4.1\left(\mathrm{qd},{ }^{1} J_{\mathrm{C}-\mathrm{P}}=5.4,{ }^{1} J_{\mathrm{C}-\mathrm{H}}=127 \mathrm{~Hz}, \mathrm{P} M e_{2}\right), 11.0\left(\mathrm{q},{ }^{1} J_{\mathrm{C}-\mathrm{H}}=126 \mathrm{~Hz}, \mathrm{C}_{5} M e_{5}\right), 26.0(\mathrm{qd}$, 
$\left.{ }^{1} J_{\mathrm{C}-\mathrm{P}}=18.9,{ }^{1} J_{\mathrm{C}-\mathrm{H}}=126 \mathrm{~Hz}, \mathrm{P} M e_{2}\right), 46.8\left(\mathrm{~d},{ }^{1} J_{\mathrm{C}-\mathrm{H}}=156 \mathrm{~Hz}, C_{6} \mathrm{H}_{6}\right), 49.1\left(\mathrm{~d},{ }^{1} J_{\mathrm{C}-\mathrm{H}}=155\right.$ $\left.\mathrm{Hz}, C_{6} \mathrm{H}_{6}\right), 92.6\left(\mathrm{~s} C_{5} \mathrm{Me}_{5}\right), 124.6 \mathrm{ppm}\left(\mathrm{dd},{ }^{3} J_{\mathrm{C}-\mathrm{P}}=6.0,{ }^{1} J_{\mathrm{C}-\mathrm{H}}=156 \mathrm{~Hz}, C_{6} \mathrm{H}_{6}\right) .{ }^{31} \mathrm{P}\left\{{ }^{1} \mathrm{H}\right\}$ NMR (109 MHz, benzene- $d_{6}, 25{ }^{\circ} \mathrm{C}$ ): $\delta 127.8 \mathrm{ppm}$. Anal. Calcd for $\mathrm{C}_{28} \mathrm{H}_{43} \mathrm{P}_{1} \mathrm{Ru}_{2}: \mathrm{C}$, 54.88; H, 7.07. Found: C, 54.45; H, 7.17. 